\title{
Relationship between incommensurability and superconductivity in Peierls distorted charge-density-wave systems
}

\author{
G. Seibold and S. Varlamov \\ Institut für Physik, BTU Cottbus, PBox 101344, 03013 Cottbus, Germany
}

(October 28, 2018)

\begin{abstract}
We study the pairing potential induced by fluctuations around a charge-density wave (CDW) with scattering vector $\mathbf{Q}$ by means of the Fröhlich transformation. For general commensurability $\mathrm{M}$, defined as $|\mathbf{k}+M \mathbf{Q}\rangle=|\mathbf{k}\rangle$, we find that the intraband pair scattering within the M subbands scales with $M$ whereas the interband pair scattering becomes suppressed with increasing CDW order parameter. As a consequence superconductivity is suppressed when the Fermi energy is located between the subbands as it is usually the case for nesting induced CDW's, but due to the vertex renormalization it can be substantially enhanced when the chemical potential is shifted sufficiently inside one of the subbands. The model can help to understand the experimentally observed dependence of the superconducting transition temperature from the stripe phase incommensurability in the lanthanum cuprates.
\end{abstract}

PACS numbers: 74.20.Fg, 74.25.-q, 74.25.Kc, 74.72.Dn

\section{INTRODUCTION}

It is now well established that some of the cuprate high- $\mathrm{T}_{c}$ superconductors show a strong depression of $T_{c}$ when the doping concentration coincides with an integer fraction. This feature was first detected in $\mathrm{La}_{2-x} \mathrm{Ba}_{x} \mathrm{CuO}_{4}$ 佂 where the superconducting transition temperature displays a clear minimum near compositions $x \approx 0.12 \approx 1 / 8$. Subsequent measurements on $\mathrm{La}_{2-x} \mathrm{Sr}_{x} \mathrm{CuO}_{4}$ (LSCO) also resulted in a detectable dip of $T_{c}$ around the same doping concentrations [2] although the reduction is less pronounced in this system than in the Ba-doped samples.. Further insight into this anomaly was provided by inelastic neutron scattering measurements on $\mathrm{La}_{2-x} \mathrm{Sr}_{x} \mathrm{CuO}_{4}$ (LSCO) [3] 5] wich revealed the existence of incommensurate spin correlations in this material. More recently it was found that these incommensurate spin fluctuations are pinned in $\mathrm{Nd}$ doped LSCO oxide compounds [6,7] leading to the appearance of magnetic and charge-order superlattice peaks in the neutron diffraction pattern. These measurements therefore provide strong evidence for the occurence of spinand charge-stripe order in the lanthanum compounds which becomes static when the lattice undergoes a transition to a low temperature tetragonal (LTT) phase as it is the case for the Ba- and Nd doped systems. However, there is now growing evidence that this structural phase transition is not a necessary condition for the occurence of static stripe order which recently has also been detected in LSCO [8] and oxygen-doped $\mathrm{La}_{2} \mathrm{CuO}_{4+y}$ [9]. It should be noted that in case of the LSCO compound, ultrasonic measurements carried out for doping $x=0.12$ show a mode softening below $\sim 45 \mathrm{~K}$ [10], which can be understood as a precursor of the LTT structural phase transition. This in turn can act as the pinning mechanism for the fluctuating stripe order and therefore explain the small dip in $T_{c}$ around $x \approx 1 / 8$ for the LSCO material.
With regard to the origin of incommensurable scattering it has been concluded that the stripe ordering is driven by the charges [6, 11] since the magnetic peaks appear at lower temperatures than the charge-order peaks, consistent with an Landau free-energy analysis of coupled charge- and spin-density order parameters [12]. For completeness we want to mention that incommensurate spin correlations have also been detected in the YBCO compounds 13 and the existence of charge modulation in the $\mathrm{CuO}_{2}$ planes of Bi2212 was proposed early on in Ref. [14].

It is therefore naturally to ask wether there may exist a connection between the occurence of stripe modulations and the superconducting pair formation. A commonly held opinion is that stripe correlations naturally lead to a depression of $\mathrm{T}_{c}$ (as observed in Nd-doped LSCO) due to the (nesting induced) reduction of the density of states at the Fermi level and the localization of the charge carriers through the pinning potential of a possible structural phase transition. However, most interesting results have been obtained in the LSCO system where both the incommensurate spin fluctuations and superconductivity have been studied as a function of doping [15]. It turned out that the incommensurability of the magnetic fluctuations (defined as the deviation of the magnetic peak from its antiferromagnetic position) scales linearly with the superconducting transition temperature $\mathrm{T}_{c}$ up to optimal doping, i.e. both $\delta$ and $T_{c}$ appear at the same critical concentration $\mathrm{x} \approx 0.05$ and follow the same doping dependence. This result strongly suggests that incommensurate stripe ordering and superconductivity may arise from the same instability as it is proposed in the framework of a Quantum Critical Point (QCP) scenario [16]. Within this model the singular scattering induced by the critical charge fluctuations would be responsible for both the anomalous normal-state properties and the large superconducting critical temperatures. Locating the QCP 
near optimal doping the phase diagram of the cuprates is partitioned in a (nearly) ordered, a quantum critical, and a quantum disordered region naturally corresponding to the under-, optimally, and over-doped regions of the phase diagram of the cuprates.

Presupposing the existence of charge stripe correlations we want to investigate in the present paper how the incommensurability of these modulations may affect the pairing potential. For convenience we restrict ourselves to the static case using a Fröhlich type transformation to study the problem how the modification of the electron states due to the formation of a charge-density wave (CDW) influences the superconducting order parameter for given (in)commensurability. It should be mentioned that a similar model has already been considered in Ref. [17] where the effective electron-electron interaction mediated by phonons has been factorized due to CDW and superconducting order parameters. However, this procedure is not consistent with the formation of a CDW since it leads to an unnatural energy cutoff for the particle-hole excitations whereas a static CDW involves particle-hole scattering processes of all available energies. The correct procedure which we will follow below is therefore first to diagonalize the CDW mean-field part of the hamiltonian and after that to calculate the effective interaction between the new quasiparticles arising from fluctuations around the frozen-in CDW. It turns out that this interaction strongly depends on the (in)commensurability $\mathrm{M}$ of the CDW with the underlying lattice which opens the possibility of increasing $\mathrm{T}_{c}$ by doping the CDW while fixing its periodicity. We want to stress that this mechanism is not due to the appearance of $1 / \sqrt{\omega}$-singularities in the density of states (DOS) but has its origin in the modification of the electron-phonon vertex function.

In Sec. II we introduce our model hamiltonian and in Sec. III we will derive the effective quasiparticle interaction in a two-dimensional CDW system of arbitrary commensurability using a Fröhlich transformation approach. Explicit results are presented in Sec. III for the simplest case of a CDW with modulation $\mathbf{Q}=(\pi, \pi)$. Despite its low commensurability it turns out that $\mathrm{T}_{c}$ is considerably enhanced due to the vertex renormalization when the chemical potential is located sufficiently inside one of the two subbands. We finally summarize our conclusions in Sec. IV.

\section{MODEL HAMILTONIAN}

Our investigations are based on the following lattice model which describes the coupling of electrons to a dispersionless phonon mode

$$
H=\sum_{k, \sigma} \epsilon_{k} c_{k, \sigma}^{\dagger} c_{k, \sigma}+\omega_{0} \sum_{q} b_{q}^{\dagger} b_{q}
$$

$$
+\frac{g}{\sqrt{N}} \sum_{k q, \sigma} c_{k+q, \sigma}^{\dagger} c_{k, \sigma}\left(b_{q}+b_{-q}^{\dagger}\right)
$$

where $c_{k, \sigma}^{(\dagger)}$ destroys (creates) an electron in the state $\mathrm{k}$ and energy $\epsilon_{k}$. The operatos $b_{q}^{(\dagger)}$ destroy (create) a phonon with momentum $q$. For convenience we take the electron-phonon matrix element $g$ and the phonon frequency $\omega_{0}$ to be constant.

Within this model the transition to a static CDW is signaled by a diverging polarizability for a certain wave vector $\mathrm{Q}$ and at some critical doping concentration. The phonon part of the CDW mean-field wave-function is usually taken to be a coherent state which means that for the selected Q-values the phonon operators are replaced by a complex number.

The coherent state contribution is extracted from the hamiltonian Eq. (11) by means of a displacement transformation $b_{q}=a_{q}-\alpha_{q}$ where the operators $a_{q}$ now correspond to the phonon fluctuations around the coherent state $\left|\alpha_{q}\right\rangle$. The CDW order parameter is defined through $g \alpha_{q} / \sqrt{N}=\Delta_{Q} \delta_{q, Q}$. We have neglected the contribution of higher harmonics to the CDW profile which are not essential for our present considerations.

We thus obtain the following hamiltonian

$$
H=H_{C D W}+H_{F l}+H_{P}
$$

where

$$
\begin{aligned}
H_{C D W} & =\sum_{k, \sigma} \epsilon_{k} c_{k, \sigma}^{\dagger} c_{k, \sigma}+N \frac{\omega_{0}}{g^{2}}\left|\Delta_{Q}\right|^{2} \\
& -\sum_{k, \sigma}\left(\Delta_{Q} c_{k+Q, \sigma}^{\dagger} c_{k, \sigma}+\Delta_{Q}^{*} c_{k-Q, \sigma}^{\dagger} c_{k, \sigma}\right)
\end{aligned}
$$

decribes the static CDW scattering with wave vector $\mathrm{Q}$ of the electrons. The essential term which contains the coupling of the phonon fluctuations to the electronic degrees of freedom corresponds to

$$
H_{F L}=\frac{g}{\sqrt{N}} \sum_{k q, \sigma} c_{k+q, \sigma}^{\dagger} c_{k, \sigma}\left(a_{q}+a_{-q}^{\dagger}\right)
$$

and

$$
H_{P}=\omega_{0} \sum_{q} a_{q}^{\dagger} a_{q}
$$

is the energy of the phonon fluctuations.

\section{EFFECTIVE INTERACTION FOR GENERAL COMMENSURABILITY}

For a given k-state in the Brillouin zone we define the commensurability $\mathrm{M}$ of the CDW as the number of scattering events in the same direction needed to return to the equivalent state, i.e. $|\mathbf{k}+M \mathbf{Q}\rangle=|\mathbf{k}\rangle$. It is clear that the larger $\mathrm{M}$ the higher is the 'incommensurability' 
of the CDW with the underlying lattice. In order to keep the comprehensibility of our presentation we consider in the following a two-dimensional system where the CDW scattering vector $\mathbf{Q}=\left(Q_{x}, 0\right)$ is along the x-direction. Within the derivation presented below other orientations would only change the k-state labeling. The reduced Brillouin zone (BZ) then is defined by $-\pi / M<k_{x} \leq \pi / M$ and $-\pi / a<k_{y} \leq \pi / a$ and the electron operators in the reduced zone read as

$$
c_{n}(k, \sigma) \equiv c_{k_{x}-\operatorname{sgn}\left(k_{x}\right)(n-1) Q_{x}, k_{y}, \sigma}
$$

where the same definition holds also for the phonon operators. The signum function has been introduced in order to obtain symmetric subbands in the reduced zone with respect to $k_{x}$. Performing a unitary transformation $c_{n}(k, \sigma)=\sum_{m=1}^{M} A_{n m} f_{m}(k, \sigma)$ the mean-field part of the hamiltonian Eq. (3) can be diagonalized

$$
H_{C D W}=\sum_{k, \sigma} \sum_{n} E_{n}(k) f_{n}^{\dagger}(k, \sigma) f_{n}(k, \sigma)
$$

where $E_{n}(k)$ denotes the dispersion of the n-th subband in the reduced BZ. It can be further shown that the condition $E_{n}\left(k_{x}, k_{y}\right)=E_{n}\left(-k_{x}, k_{y}\right)$ requires for the transformation matrices the property $A_{n m}\left(k_{x}, k_{y}\right)=$ $A_{n m}^{*}\left(-k_{x}, k_{y}\right)=A_{m n}^{-1}\left(-k_{x}, k_{y}\right)$.

The coupling term Eq. (4) rewritten in terms of the new quasiparticle operators reads as

$$
\begin{aligned}
H_{F L} & =\frac{g}{\sqrt{N}} \sum_{k q, \sigma} \sum_{s t m} \Gamma_{s t m}(k, q) f_{s}^{\dagger}(\widetilde{k+q}, \sigma) f_{t}(k, \sigma) \\
& \times\left(a_{m}(q)+a_{m}^{\dagger}(-q)\right)
\end{aligned}
$$

where we have introduced the vertex function

$$
\Gamma_{s t m}(k, q)=\sum_{n} A_{\mathcal{N}, s}^{*}(\widetilde{k+q}) A_{n, t}(k)
$$

and the index $\mathcal{N}=\mathcal{N}(n, m, k, q)$ is defined below. Unfortunately at this point the notation is getting complex both due to the addition of momenta in the signum function and the scattering of electrons between neighboring reduced zones. For this reason the following definitions have been made in Eqs. (8, 9)

$$
\begin{aligned}
& \text { For } \operatorname{sgn}\left(k_{x}\right)=\operatorname{sgn}\left(q_{x}\right) \text { and }\left|k_{x}+q_{x}\right|<\pi / M: \\
& \widetilde{k+q} \equiv k+q ; \quad \mathcal{N}=n+m-1 \\
& \text { For } \quad \operatorname{sgn}\left(k_{x}\right) \neq \operatorname{sgn}\left(q_{x}\right) \text { and }\left|k_{x}+q_{x}\right|<\pi / M: \\
& \widetilde{k+q} \equiv k+q ; \quad \mathcal{N}=\operatorname{sgn}\left(\left|k_{x}\right|-\left|q_{x}\right|\right)(n-m)+1 \\
& \text { For } \quad \operatorname{sgn}\left(k_{x}\right)=\operatorname{sgn}\left(q_{x}\right) \quad \text { and } \quad\left|k_{x}+q_{x}\right|>\pi / M: \\
& \widetilde{k+q} \equiv k+q-\operatorname{sgn}\left(k_{x}+q_{x}\right) Q_{x} ; \quad \mathcal{N}=n+m-2
\end{aligned}
$$

The coupling term Eq. (8) can now be eliminated in first order by means of a standard Fröhlich transformation (see e.g. 18), i.e. we expand the hamiltonian Eq.
(2) in a commutator series up to second order in the vertex $\Gamma$

$$
\left.\tilde{H}=\mathrm{e}^{-S} H \mathrm{e}^{S}=H+[H, S]+\frac{1}{2}[H, S], S\right]+\cdots
$$

with $\mathrm{S}$ being specified by the condition:

$$
\left[H_{C D W}+H_{P}, S\right]=-H_{F l}
$$

Representing $\mathrm{S}$ in the form

$$
\begin{aligned}
S & =\frac{1}{\sqrt{N}} \sum_{k q, \sigma} \sum_{u v w} f_{u}^{\dagger}(\widetilde{k+q}, \sigma) f_{v}(k, \sigma) \\
& \times\left[P_{u v w}^{-}(k, q) a_{w}(q)+P_{u v w}^{+}(k, q) a_{w}^{\dagger}(-q)\right]
\end{aligned}
$$

the requirement Eq. (14) is satisfied by

$$
P_{u v w}^{ \pm}(k, q)=-g \frac{\Gamma_{u v w}(k, q)}{E_{u}(\widetilde{k+q})-E_{v}(k) \pm \omega_{0}}
$$

We thus finally obtain a transformed hamiltonian which, among terms describing the renormalization of phonon and quasiparticle energies, contains an effective quasiparticle interaction. The relevant contribution for superconductivity consists of those terms which describe the scattering of a zero momentum pair between the subbands of the CDW system and reads as

$$
\begin{aligned}
V_{S C} & =\frac{1}{N} \sum_{k q, \sigma} \sum_{s t m}\left|\Gamma_{s t m}(k, q)\right|^{2} \frac{g^{2} \omega_{0}}{\left[E_{s}(\widetilde{k+q})-E_{t}(k)\right]^{2}-\omega_{0}^{2}} \\
& \times f_{s}^{\dagger}(\widetilde{k+q}, \sigma) f_{s}^{\dagger}(\widetilde{k-q},-\sigma) f_{t}(-k,-\sigma) f_{t}(k, \sigma)
\end{aligned}
$$

Obviously this expression reduces to the well-known phonon mediated electron-electron interaction in the limit $\Delta_{Q} \rightarrow 0$ when the transformation matrices are given by $A_{n m}(k)=\delta_{n, m}$ and the vertex contribution becomes independent of the band indices $\sum_{m}\left|\Gamma_{s t m}(k, q)\right|^{2} \rightarrow 1$. However, in case of a well developped CDW the transformation takes the form

$$
\begin{aligned}
A_{n p}(k) & \approx \frac{1}{\sqrt{M}} \exp \left(i n p \operatorname{sgn}\left(k_{x}\right)\right) \\
p & =-\pi / M \ldots \pi / M
\end{aligned}
$$

which for the vertex function leads to $\Gamma_{s t m}(k, q)=$ $\exp (i \phi(m)) \delta_{s, t}$ i.e. the phonon branch-index m only enters through a phase $\phi(m)$ depending on the three ranges defined in Eqs. (10 12). As a consequence the vertex contribution

$$
\sum_{m}\left|\Gamma_{s t m}(k, q)\right|^{2}=M \delta_{s, t}
$$

now restricts the pair-pair scattering to the individual subbands and the effective interaction becomes proportional to the commensurability $M$ of the CDW. Since the number of states within each subband decreases with 
the commensurability as $2 N / M$ it is obvious that the vertex renormalization can only lead to an effective enhancement of the quasiparticle interaction as long as the cutoff range of its attractive contribution lies within one of the subbands. For the present two-dimensional model with a phonon mediated interaction this means that for $M<B / \omega_{D}$ the CDW formation can lead to a substantial enhancement of the superconducting order parameter where $\mathrm{B}$ is the bandwidth and $\omega_{D}$ denotes the Debye frequency as a characteristic energy scale for the cutoff. Of course the considered limit of a large CDW order parameter is rather academic since then the quasiparticles become localized and superconductivity will be suppressed. However, the essential point is that with the onset of CDW scattering the intraband pairing is amplified with a simultaneous decrease of the interband pairing amplitude. Both processes do not compensate since most of the interband contribution to the interaction falls out of the range of attraction.

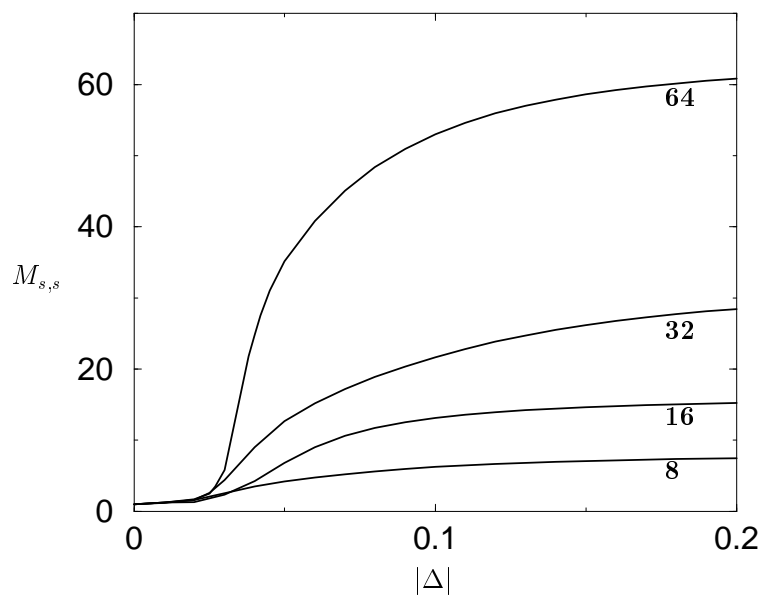

FIG. 1. Intraband vertex contribution to the effective interaction $M_{s, s}=\sum_{m}\left|\Gamma_{s s m}(k, q)\right|^{2}$ for $\mathbf{Q}=(3 \pi / 4,0),(5 \pi / 8,0)$, $(9 \pi / 16,0)$, and $(19 \pi / 32)$ corresponding to the commensurabilities $M=8,16,32$, and 64 as indicated in the figure. $\mathbf{k}=(\pi / 80, \pi), \mathbf{q}=(-\pi / 40,0)$

In the remainder of this section we consider as an example the CDW induced vertex renormalization for holes with energy dispersion

$$
\begin{aligned}
\epsilon_{k} & =2 t_{1}\left(\cos k_{x}+\cos k_{y}\right)+4 t_{2} \cos k_{x} \cos k_{y} \\
& +2 t_{3}\left(\cos 2 k_{x}+\cos 2 k_{y}\right)
\end{aligned}
$$

where we use the hopping integrals $t_{1}=45 \mathrm{meV}, t_{2}=$ $5 \mathrm{meV}, t_{3}=16 \mathrm{meV}$ 20. For small and moderate hole concentrations this dispersion gives rise to an open (i.e. centered around $(\pi, \pi)$ ) Fermi surface as observed in various photoemission experiments in the high- $\mathrm{T}_{c}$ cuprates (see e.g. [19] and references therein). Moreover one finds [20] that in the 'underdoped' regime this model leads to a polarizability which is peaked along the $k_{x^{-}}$and $k_{y^{-}}$axis of the Brillouin zone repectively. As a consequence the system becomes unstable towards a vertically oriented CDW when the electron-phonon coupling constant exceeds some critical value. In the following we consider a scattering vector $Q \approx(0.6 \pi, 0)$ which in our model is realized for hole concentrations $\delta \approx 0.25$.

Fig. 1 displays the intraband vertex contribution to the effective interaction $M_{s, s}(k, q)=\sum_{m}\left|\Gamma_{s s m}(k, q)\right|^{2}$ for different commensurabilities, i.e. the scattering vector $\mathbf{Q} \approx(0.6 \pi, 0)$ is approximated by increasing fractions $(n / m, 0)$ as indicated in the figure capture. For each case we have selected a subband which covers the Fermi level and the phase of the CDW order parameter is fixed to $\phi=\pi / 3$. The general behavior of $M_{s, s}(k, q)$ is consistent with the limiting values discussed above. Starting from $M_{s, s}(k, q)=1$ one observes a strong increase for some critical value of the CDW order parameter $|\Delta|_{\text {crit }}$ and a saturation to the maximum commensurability in the limit of large $|\Delta|$. The value of $|\Delta|_{\text {crit }}$ strongly depends on the selected band and especially on the values of $\mathbf{k}$ and $\mathbf{q}$. When these states are choosen such that their scattered states (i.e. $\mathbf{k}+\mathbf{Q}, \mathbf{k}+2 \mathbf{Q} \ldots$ and $\mathbf{q}+\mathbf{Q}$, $\mathbf{q}+2 \mathbf{Q} \ldots)$ do not differ much in energy, the onset of the increase of $M_{s, s}(k, q)$ occurs for smaller $|\Delta|$ as in case of large energy differences between the scattered states.
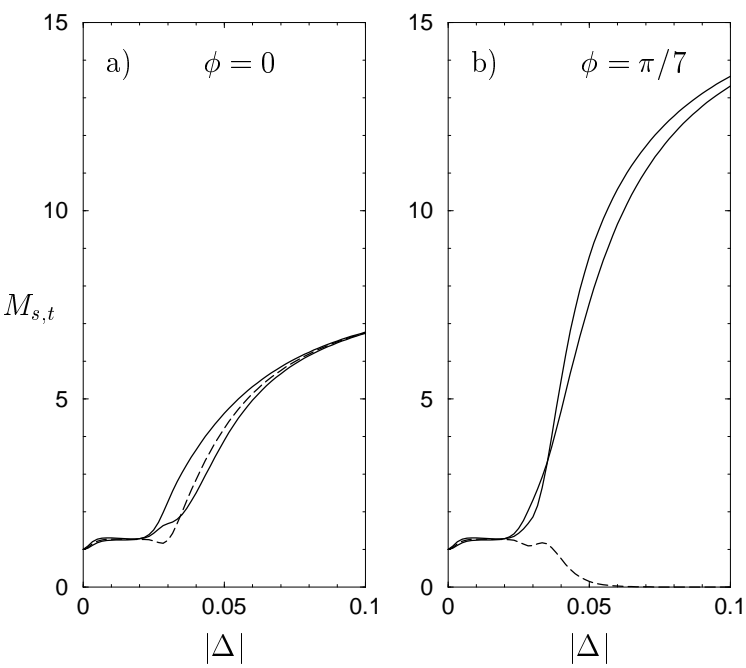

FIG. 2. Vertex contribution to the effective interaction $M_{s, t}=\sum_{m}\left|\Gamma_{s t m}(k, q)\right|^{2}$ for two energetically neighbored subbands of a CDW with scattering vector $\mathbf{Q}=(5 \pi / 8,0)$ (commensurability $\mathrm{M}=16$ ). The solid lines correspond to the intraband scattering $\left(M_{1,1}\right.$ and $\left.M_{2,2}\right)$ whereas the dashed line refers to the scattering between the subbands $\left(M_{1,2}\right)$. a) and b) are plots for two different phases $\phi=0, \pi / 7$ of the CDW order parameter $\Delta=|\Delta| \exp (i \phi)$.

Another point not discussed so far is the influence of the phase of the CDW order parameter. In Fig. 2 we show the vertex contribution $M_{s, t}$ for two energetically neighbored subbands of a CDW with scattering vector 
$Q=(5 \pi / 8,0)$ (commensurability $\mathrm{M}=16)$ and two different phases $\phi=0, \pi / 7$. In case of $\phi=0$ not only the intraband scattering is enhanced with increasing $\Delta$ but also the scattering between the bands due to their large degeneracy. As a result the curves for the intraband contribution approach half the value of the commensurability only in the limit of large $|\Delta|$, in favor of a similar behavior of the interband renormalization. As shown in Fig. 2b this interband scattering can be suppressed by choosing a different phase which essentially restores the 'orthogonality' between the quasi-degenerate states. However, for the quasiparticle pairing the phase of the CDW order parameter is not relevant since it essentially redistributes the pair-scattering between different channels each having the same pairing amplitude.

\section{CDW WITH COMMENSURABILITY M=2}

We will now discuss the consequences of the renormalized vertex to superconductivity in more detail studying the simplest available CDW system in two dimensions with scattering vector $Q=(\pi, \pi)$ and electron dispersion $\epsilon_{k}=-\epsilon_{k+Q}=-B / 2\left(\cos k_{x}+\cos k_{y}\right)$, corresponding to nearest neighbor hopping of particles on a square lattice. The hamiltonian Eq. (3) then can be easily diagonalized and one obtains

$$
\begin{aligned}
H_{\mathrm{CDW}} & =\sum_{k, \sigma} E_{k}\left[f_{1}^{\dagger}(k, \sigma) f_{1}(k, \sigma)-f_{2}^{\dagger}(k, \sigma) f_{2}(k, \sigma)\right] \\
& +\frac{N}{4} \frac{\omega_{0}}{g^{2}} \Delta_{\mathrm{CDW}}^{2}
\end{aligned}
$$

where the dispersion of the CDW system is given by $E_{k}=\sqrt{\Delta_{\mathrm{CDW}}^{2}+\epsilon_{k}^{2}}$.

Following the procedure described above we then derive the pairing hamiltonian

$$
\begin{aligned}
H_{\mathrm{el}-\mathrm{el}} & =\frac{1}{N} \sum_{k q, \sigma} \sum_{i, j}^{2} \Gamma_{i j}^{2}(k q) \frac{g^{2} \omega_{0}}{[E(k+q)-E(k)]^{2}-\omega_{0}^{2}} \\
& \times f_{i}^{\dagger}(-k-q,-\sigma) f_{i}^{\dagger}(k+q, \sigma) f_{j}(k, \sigma) f_{j}(-k,-\sigma)
\end{aligned}
$$

where the vertex contribution reads

$$
\Gamma_{i j}^{2}(k q)=\left(1 \pm \frac{\Delta_{\mathrm{CDW}}^{2}}{E_{k} E_{k+q}}\right)
$$

and the $+(-)$ sign corresponds to $i=j(i \neq j)$. The pair scattering between valence and conduction band thus is decreased with increasing CDW order parameter in favor of an enhancement of the intraband pairing amplitude.

We have calculated selfconsistently the CDW- and SC order parameters within a BCS decoupling scheme and furthermore have used a BCS-type approximation for the interaction in Eq. (21), i.e. replacing $g^{2} \omega_{0} /[E(k+q)-E(k)]^{2}-\omega_{0}^{2}$ by a constant potential
$-V$ for k-states out to a cutoff energy $\omega_{D}$. The symmetry of the SC gap is then completely determined by the symmetry of $\Gamma_{i j}^{2}(k q)$ and takes the form

$$
\Delta_{S C}(k)=\Delta_{0}+\alpha \frac{\Delta_{\mathrm{CDW}}^{2}}{E_{k}} .
$$

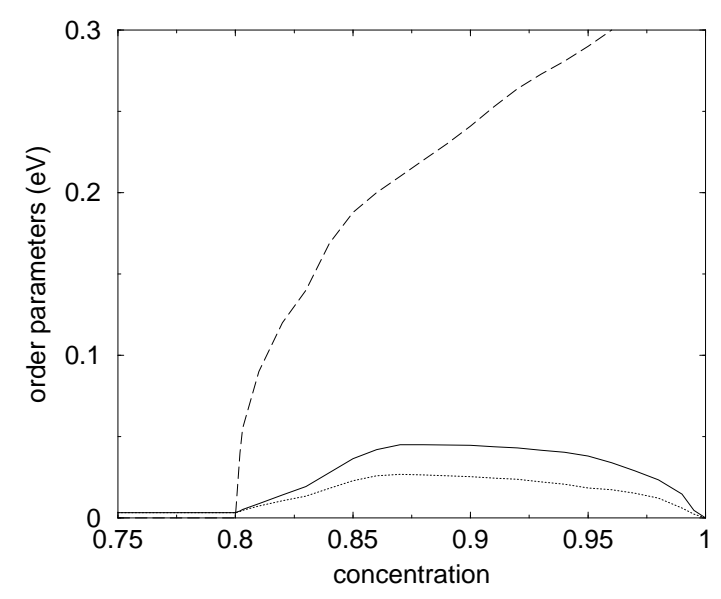

FIG. 3. CDW- and SC order parameters as a function of doping for a $Q=(\pi, \pi)$ CDW system. Dashed line: CDW gap $\left(\Delta_{\mathrm{CDW}}\right)$, Dotted line: constant part of the SC gap $\left(\Delta_{0}\right)$, Solid: Maximum value of the total SC gap $\left(\Delta_{S C}^{\max }(k)\right)$. For convenience the calculation has been performed for a constant DOS $\rho(\omega)=1 /(2 B) \Theta\left(B^{2}-\omega^{2}\right)$. Parameters: $\mathrm{B}=0.8 \mathrm{eV}$, $\mathrm{V}=0.25 \mathrm{eV}, \omega_{D}=0.04 \mathrm{eV}$.

Fig. 3 displays the results as a function of doping (concentration $n=1$ corresponds to half-filling) for a selected parameter set. The onset of CDW scattering leads to a $1 / \sqrt{\omega}$ contribution to the DOS around the CDW gapedges. As a consequence also for concentrations $n<1$ the DOS at the Fermi energy is increased leading to an enhancement of the constant part of the SC gap. Besides this well known effect it can be seen that the vertex renormalization induces a considerable increase of the ) $\mathrm{SC}$ order parameter due to the amplification of the intraband pairing. Additionally superconductivity is suppressed when the concentration leads to perfect nesting of the Fermi surface (i.e. $n=1$ ). In the small coupling limit one can obtain an explicit expression for the vertex contribution to the SC gap under the assumption that the chemical potential $\mu$ is sufficiently inside the lower subband $\left(\mu<-\Delta_{\mathrm{CDW}}-\omega_{D}\right)$. We find

$$
\alpha=-\frac{V}{4 B|\mu|} \Delta_{0} \ln \left[\frac{\Delta_{0}^{2}}{4}\left(1 / \omega_{D}^{2}+1 / \mu^{2}\right)\right]
$$

where B denotes the electronic bandwidth. Note that $\alpha>0$ since the expression has been obtained under the assumption $\Delta_{0}<\omega_{D},|\mu|$. 


\section{CONCLUSION}

To summarize, we have shown that the vertex renormalization can lead to a substantial enhancement of the superconducting order parameter in a Peierls distorted CDW system when the chemical potential is located sufficiently inside one of the subbands. Since for a nesting induced instability the Fermi level always falls in the DOS minimum between two subbands it is clear that this mechanism can only work when the CDW modulation is fixed by some external (e.g. lattice-) potential while the carrier concentration is varied. This in turn, however, is not in favor of superconductivity due to the localization of the charge carriers. The more promising alternative would be a nesting independent CDW instablity as realized for example in a frustrated phase separation scenario 16. In this case the CDW formation occurs due to the interplay between long-range Coulomb forces and a phase separation instability and is not related to any Fermi wave vector $k_{F}$.

Our considerations provide a possible explanation for the linear relationship between $T_{c}$ and incommensurability as observed in the underdoped lanthanum cuprates [15]. In these systems the incommensurability $M$ is related to the deviation of the magnetic scattering vector $\mathbf{Q}=(\pi / M, \pi)$ from $(\pi, \pi)$, i.e. upon doping towards $T_{c}^{\max }$ one observes an increase of the incommensurability M. According to our findings this in turn induces an enhancement of the effective interaction $V_{\text {eff }} \sim M$ which causes $\mathrm{T}_{c}$ to scale with the incommensurability $\mathrm{M}$. Of course we have used a very simplified model restricting ourselves to static CDW formation and neglecting spin degrees of freedom. However the main outcome of our investigations, namely the scaling of the effective interaction with the commensurability, should hold also in case of dynamic CDW scattering. Indeed there is experimental evidence that in the $\mathrm{La}_{2-x} \mathrm{Sr}_{x} \mathrm{CuO}_{4}$ system, where no pinning is provided by the LTO-LTT transition, stripes fluctuate very slowly [21] allowing for an order parameter description in principle also in this case.

\section{ACKNOWLEDGMENTS}

We would like to thank A. Bill for helpful comments and a critical reading of the manuscript. G.S. greatfully acknowledges stimulating discussions with M. Grilli, C. Castellani and C. Di Castro at the early stage of this work.
[1] A. R. Moodenbaugh, Y. Xu, M. Suenaga, T. J. Folkerts, and R. N. Shelton, Phys. Rev. B38, 4596 (1988).

[2] H. Takagi, T. Ido, S. Ishibashi, M. Uota, S. Uchida, and Y. Tokura, Phys. Rev. B 40, 2254 (1989).

[3] S.-W. Cheong et al., Phys. Rev. Lett. 67, 1791 (1991).

[4] T. E. Mason, G. Aeppli, and H. A. Mook, Phys. Rev. Lett. 65, 2466 (1990).

[5] T. R. Thurston et al., Phys. Rev. B46, 9128 (1992).

[6] J. M. Tranquada, B. J. Sternlieb, J. D. Axe, Y. Nakamura, and S. Uchida, Nature 375, 561 (1995).

[7] J. M. Tranquada, J. D. Axe, N. Ichikawa, A. R. Moodenbaugh, Y. Nakamura, and S. Uchida, Phys. Rev. Lett. 78, 338 (1997).

[8] H. Kimura, K. Hirota, H. Matsushita, K. Yamada, Y. Endoh, S. H. Lee, C. F. Majkrzak, R. W. Erwin, G. Shirane, M. Greven, Y. S. Lee, M. A. Kastner, and R. J. Birgeneau, Phys. Rev. B 59, 6517 (1999).

[9] Y. S. Lee, R. J. Birgeneau, M. A. Kastner, Y. Endoh, S. Wakimoto, K. Yamada, R. W. Erwin, S.-H. Lee, and G. Shirane, cond-mat/9902157.

[10] T. Suzuki, T. Goto, K. Chiba, T. Shinoda, and T. Fukase, H Kimura, K. Yamada, M. Ohashi, and Y. Yamaguchi, Phys. Rev. B 57, 3229 (1998).

[11] T. Niemöeller, H. Hühnefeld, J. R. Schneider, N. Ichikawa, S. Uchida, T. Frello, N. H. Andersen, J. M. Tranquada, cond-mat/9904383.

[12] O. Zachar, S. A. Kivelson, and V. J. Emery, Phys. Rev. B 57, 1422 (1998).

[13] H. A. Mook, P. Dai, S. M. Hayden, G. Aeppli, T. G. Perring, and F. Dogan, Nature 395, 580 (1998).

[14] A. Bianconi, Proceedings of the workshop on Phase Separation In Cuprate Superconductors, edited by K. A. Müller and G. Benedek (World Scientific, Singapore, 1993).

[15] K. Yamada, C. H. Lee, K. Kurahashi, J. Wada, S. Wakimoto, S. Ueki, H. Kimura, Y. Endoh, S. Hosoya, G. Shirane, R. J. Birgeneau, M. Greven, M. A. Kastner, and Y. J.Kim, Phys. Rev. B 57, 6165 (1998).

[16] C. Castellani, C. Di Castro, and M. Grilli, Phys. Rev. Lett. 75, 4650 (1995); C. Castellani, C. Di Castro, and M. Grilli, Z. für Physik, 103, 137 (1997).

[17] C. A. Balseiro and L. M. Falicov, Phys. Rev. B 20, 4457 (1979).

[18] M. Wagner, Unitary Transformations In Solid State Physics, (North-Holland, Amsterdam 1986).

[19] Z.-X. Shen and D. S. Dessau, Phys. Rep. 253, 1 (1995).

[20] I. Eremin, M. Eremin, S. Varlamov, D. Brinkmann, M. Mali, and J. Roos, Phys. Rev. B 56, 11305 (1997); I. Eremin, M. Eremin, and S. Varlamov, Proceedings of the Stripes'98 conference,Rome, Italy, J. Supercond., to be published.

[21] A. W. Hunt, P. M. Singer, K. R. Thurber, and T. Imai, cond-mat/9902348. 